\title{
EPIDEMIOLOGÍA DEL TIZÓN TARDÍO [Phytophthora infestans (MONT.) DE BARY] EN QUINCE INTRODUCCIONES DE TOMATE SILVESTRE
}

\section{EPIDEMIOLOGY OF LATE BLIGHT [Phytophthora infestans (MONT.) DE BARY] IN FIFTEEN INTRODUCTIOS OF WILD TOMATO}

\author{
Luisa Fernanda Cardona-Piedrahita ${ }^{1}$, Jairo Castaño-Zapata ${ }^{2}$, Nelson Ceballos-Aguirre ${ }^{3}$
}

\begin{abstract}
${ }^{1}$ Magister en Fitopatología, Programa de Maestría, Facultad Ciencias Agropecuarias. Universidad de Caldas, e-mail: luisafernandacardona@gmail.com; ${ }^{2}$ Ph.D. en Fitopatología, Profesor Titular, Facultad Ciencias Agropecuarias. Universidad de Caldas, e-mail: jairo.castano_z@ucaldas.edu.co; ${ }^{3}$ Doctorado en Ciencias Agrarias, Profesor Asistente, Facultad Ciencias Agropecuarias. Universidad de Caldas, Calle 65 No. 26-10, Manizales-Colombia, e-mail: nelson.ceballos@ucaldas.edu.co
\end{abstract}

Rev. U.D.C.A Act. \& Div. Cient. 19(1): 45-54, Enero-Junio, 2016

\section{RESUMEN}

La mayoría de los genes responsables de la resistencia en tomate a hongos, bacterias, virus y nematodos fitoparásitos han sido derivados de especies silvestres. Es así, como se deben evaluar e identificar genotipos silvestres promisorios, por su respuesta a los principales problemas patológicos de la especie cultivada, para considerarlos en programas de mejoramiento del tomate. Por consiguiente, es necesario evaluar la resistencia de introducciones silvestres del tomate al tizón tardío, causado por Phytophthora infestans, la enfermedad más devastadora en este cultivo. El estudio, se realizó en la granja Montelindo de la Universidad de Caldas, en quince introducciones de tomate silvestre, bajo dos sistemas de producción: libre exposición y semitecho. El diseño experimental fue parcelas divididas; las parcelas principales fueron los dos sistemas de producción; las subparcelas, las introducciones de tomate con cuatro repeticiones. Las variables evaluadas fueron porcentaje de severidad, tasa de desarrollo (r) y área bajo la curva del desarrollo (ABCDE) del tizón tardío; asimismo, se calcularon los coeficientes de regresión de las variables analizadas. Finalmente, se realizó análisis de varianzas y pruebas de promedio tipo Duncan. De acuerdo a los análisis, bajo semitecho, se obtuvieron los valores numéricos más bajos de severidad final, r y ABCDE, con 47\%, 0,09 y 1.133 , respectivamente, sobresaliendo las introducciones LA1480, IAC1686, LA2076, LA2131 y LA2692; a libre exposición, se obtuvo los más altos valores de severidad final, $r$ y ABCDE, con $77 \%, 0,15$ y 1.573 , respectivamente; sin embargo, LA1480 y LA2076 tuvieron los valores más bajos en este sistema de producción.
Palabras clave: Chromista, recursos fitogenéticos, severidad, solanácea, tasa de desarrollo, área bajo la curva del desarrollo de la enfermedad.

\section{SUMMARY}

Most of the genes responsible of resistance of tomato to fungi, bacteria, virus and nematodes, has been derivate from wild species of tomato. Hence, it is necessary to evaluate and identify promising wild genotypes to observe their reaction to the main pathological problems of cultivated species, in order to be considered in future programs of tomato production improvement. To reach this purpose, it is necessary to carry out epidemiological studies and evaluate wild introductions against tomato late blight caused by Phytophthora infestans, the most devastating disease worldwide. The study was conducted with fifteen wild tomato introductions at the Montelindo's farm of the Universidad de Caldas, in two production systems: free exposition and semi cover. Split plots was the experimental design used, being the main plots the production systems, and the subplots, the tomato introductions, with four replications. The evaluated variables were: severity (\%), rate of disease development (r) and area under the disease progress curve (AUDPC) of late blight. In addition, the regression coefficients of the evaluated variables was calculated. Finally, an analysis of variance and Duncan's tests was conducted. According to the analysis, under the semi cover system the lowest numerical values for final severity, $\mathrm{r}$ and AUDPC, with 47\%, 0.09 and 1,133, was obtained, respectively; standing out the introductions LA1480, IAC1686, LA2131 and LA2692. In the free exposition system the highest values of these parameters with $77 \%$, 
0.15 and 1,573, respectively, were obtained; however, the introductions LA1480 and LA2075 showed the lowest values under this production system.

Key words: Chromista, phylogenetic resources, severity, solanaceae, rate of disease development, area under the disease progress curve.

\section{INTRODUCCIÓN}

El tomate (Solanum lycopersicum L.) constituye el 30\% de la producción hortícola mundial y, en Colombia, es la hortaliza más importante. Para el 2014, la producción de tomate fue de $173.580 t$, con un área de 7.467ha y un rendimiento de 23,2t/ha (DANE- ENA, 2014).

Uno de los avances tecnológicos más importantes de los seres humanos es la domesticación de las especies vegetales, para la producción de comida y otros usos industriales, como fibras, resinas, materias primas, ornamentales, entre otros. Se ha utilizado secuenciación de alto rendimiento, para identificar cambios en la secuencia de ADN y la expresión de genes, que diferencian al tomate cultivado y sus parientes silvestres. También, se han identificado cientos de genes candidatos y se han desarrollado nuevas secuencias de proteínas o han cambiado los niveles de expresión, en respuesta a la selección natural, en parientes silvestres de tomate (Koenig et al. 2013).

El tomate es susceptible a muchas enfermedades, como las causadas por Phytophthora infestans (Mont.) de Bary, Alternaria solani (Ell. \& Mart.) Jones \& Grout y Ralstonia solanacearum Smith, que son algunos de los patógenos más conocidos, por las pérdidas entre el 40 y $70 \%$ que ocasionan en las solanáceas (Chavarro \& Ángel, 2005).

El tizón tardío es causado por el Chromista Phytophthora infestans, común en zonas con temperaturas entre 15 y $22^{\circ} \mathrm{C}$ y humedad relativa mayor al $80 \%$. El patógeno, se transmite a través de semillas de tomate y puede sobrevivir en forma de micelio en otras plantas cultivadas o arvenses de la familia de las solanáceas, o en residuos de cosecha, que permanecen en el suelo (Jaramillo et al. 2007). La infección en el fruto puede oscilar del 41 al 100\% en los campos sin aplicación de fungicidas y del 12 al $65 \%$, en parcelas protegidas con fungicidas sistémicos; en el 2009, en Estados Unidos, las pérdidas totales de rendimiento en los tomates frescos y procesamiento llegaron a US\$46 y US\$66 millones, respectivamente (Nowicki et al. 2012).

La resistencia genética es la capacidad que tiene el hospedante de contrarrestar la acción de un patógeno; esta es la estrategia más ampliamente recomendada y estudiada en el manejo integrado de plagas (Lemus, 2009; Kogan, 1990).
Los parientes silvestres son una fuente vital de genes de resistencia contra enfermedades, como el tizón tardío, lo cual, ayuda a asegurar que exista una adecuada diversidad genética en el patrimonio genético, de un cultivo en particular (Lane, 2006).

La epidemiologia estudia el desarrollo de enfermedades en poblaciones de plantas; describe cómo las enfermedades de las plantas se desarrollan a través del tiempo y contempla modelos matemáticos, que pueden ayudar a tomar decisiones de manejo (Arneson, 2001). Claro ejemplo de modelos matemáticos, los describen Tatarowska et al. (2011), quienes indican que la estimación de la severidad del tizón tardío en el campo se puede hacer mediante evaluación visual de la enfermedad, estimando el área afectada en el follaje en porcentaje (Colon et al. 2004) o a través de una escala de nueve grados (Pietkiewicz, 1972). Con los datos obtenidos, se calculan parámetros epidemiológicos, como la tasa de desarrollo de la enfermedad (r) (Castaño-Zapata, 2002) o el área bajo la curva de desarrollo de la enfermedad (ABCDE) (Fry, 1978), que refleja la intensidad de la enfermedad.

Esta investigación tuvo como objetivo realizar un estudio epidemiológico de severidad, tasa de desarrollo de la enfermedad y áreas bajo la curva de desarrollo del tizón tardío, en quince introducciones de tomate silvestres, en dos sistemas de producción, con el fin de generar aportes que sirvan de soporte a futuros proyectos en el campo molecular y mejoramiento genético del tomate.

\section{MATERIALES Y MÉTODOS}

La investigación, se llevó a cabo en la granja Montelindo, propiedad de la Universidad de Caldas, situada en la vereda Santágueda, municipio de Palestina, Caldas, con temperatura media de $22,8^{\circ} \mathrm{C}$, altitud de $1.010 \mathrm{msnm}$, precipitación anual de $2.200 \mathrm{~mm}$ y humedad relativa promedio del $76 \%$ (FNC \& CENICAFÉ, 2010).

Los sistemas de producción empleados correspondieron a: libre exposición, con tutorado en guaduas, a una distancia de $3 \mathrm{~m}$ y una distancia entre surcos de $1,5 \mathrm{~m}$; también, se utilizó una cobertura de plástico tipo "mulch" tipo negro-negro, calibre 1,2 y, bajo semitecho, igual al anterior sistema, pero además provisto de una cubierta de plástico tipo Agroclear, calibre 6 .

Se evaluaron quince introducciones de tomate del banco de germoplasma de la Universidad Nacional de Colombia, sede Palmira. Diez de las quince introducciones eran procedentes del Instituto Agronómico de Campiñas, en Brasil, identificadas así: IAC391, IAC424, IAC426, IAC445, IAC1621, IAC1624, IAC1688, IAC412, IAC421 e IAC1686; las otra cinco introducciones eran procedentes del Tomato Genetics 
Resources Center, de la Universidad de California, en Estados Unidos, identificadas como: LA2076, LA1480, LA2131, LA2640 y LA2692. Como testigo comercial, se empleó la variedad Sweet million, que es susceptible al patógeno.

La siembra, se realizó en bandejas de germinación de 72 lóculos, con sustrato turba sphagnum grado 3. Pasados 30 días, se realizó el trasplante de las plántulas, cuando habían alcanzado cuatro hojas verdaderas. La distancia de siembra fue de $1,50 \mathrm{~m}$ entre surcos y $0,50 \mathrm{~m}$ entre plantas, para una densidad de población de 13.333 plantas/ha.

Para la obtención del inóculo, se sembraron tubérculos-semillas de papa criolla, variedad Yema de Huevo (Solanum phureja Juz. et Buk), en la granja Tesorito, de la Universidad de Caldas, a $2.340 \mathrm{msnm}, 17,5^{\circ} \mathrm{C}, 78 \%$ de humedad relativa y $2.000 \mathrm{~mm}$ de precipitación. El tamaño de las parcelas fue de $4 \mathrm{~m}$ de largo por $1,8 \mathrm{~m}$ de ancho, con un marco de plantación de $0,6 \mathrm{~m}$, en surcos dobles, $1,2 \mathrm{~m}$ entre calles y $0,35 \mathrm{~m}$ entre plantas. La siembra, se efectuó de manera escalonada, una cada 20 días, para un total de cinco siembras, para garantizar una fuente de inóculo constante del patógeno, con la que se obtuvo la infección artificial de las introducciones de tomate cereza.

Un día antes de la inoculación, se recolectaron las hojas más afectadas de las plantas de papa criolla, almacenándolas en cámara húmeda, para mantener activos los esporangios del patógeno. Las hojas fueron llevadas a la granja Montelindo, donde, posteriormente, se les realizó un lavado con agua destilada, a través de un tamiz, sobre una máquina fumigadora de espalda Royal Cóndor de 20L, para que los esporangios de $P h$. infestans se desprendieran y quedaran suspendidos en el agua; finalmente, se realizó la inoculación sobre las introducciones a evaluar, 41 días después de trasplante (2 meses y medio de edad), dando un cubrimiento total en el área foliar de las cuatro plantas, de cada introducción de tomate. La concentración, se midió a través de un hemacitómetro marca Bright Line, obteniendo un valor de 44.000 esporangios/mL de agua.

Se utilizó un diseño de parcelas divididas; las parcelas principales fueron asignadas a los dos sistemas de producción: libre exposición y semitecho, mientras que las subparcelas fueron asignadas a las introducciones de tomate, con cuatro repeticiones. El tamaño efectivo de la unidad experimental fue de cuatro plantas.

\section{Variables evaluadas:}

Severidad (\%) del tizón tardío. Se evaluaron las primeras cuatro hojas de las plantas que presentaban los síntomas típicos de la enfermedad, como manchas de color café con apariencia húmeda y en el envés de las hojas, con estructuras de color blanquecino, en el centro de las lesiones correspondientes a la esporulación de Ph. infestans. Estas hojas se marcaron para su seguimiento y se evaluaron en intervalos de cuatro días, usando el diagrama de severidad, elaborado por James et al. (1971), quienes ilustran severidades del 1, 10, 25 y 50\%; mediante extrapolación, se continuó registrando la severidad hasta el $100 \%$ en toda la planta. La evaluación, se realizó durante 49 días después de la inoculación.

Tasa de desarrollo (r) del tizón tardío. Para tal fin, se tomaron los valores promedios de severidad de las cuatro hojas evaluadas en porcentaje, siguiendo la metodología descrita por Castaño-Zapata (2002) y aplicando la siguiente fórmula, derivada del modelo logístico de Van der Plank (1963):

$$
\mathbf{r}=\frac{1}{\mathrm{~T}_{1}-\mathrm{T}_{0}}\left(\log _{\mathrm{e}} \frac{\mathrm{X}_{1}}{1-\mathrm{X}_{1}}-\log _{\mathrm{e}} \frac{\mathrm{X}_{0}}{1-\mathrm{X}_{0}}\right)
$$

En donde: $\mathbf{r}=$ Tasa de desarrollo; $\mathrm{T}_{1}=$ Tiempo final; $\mathrm{T}_{0}=$ Tiempo inicial; $\mathrm{X}_{1}=$ Proporción de la enfermedad en el tiempo final; $X_{0}=$ proporción de la enfermedad en el tiempo inicial.

Área bajo la curva del desarrollo de la enfermedad (ABCDE). Para ello, se tomaron los valores promedios de severidad (\%) de las cuatro hojas evaluadas por planta, siguiendo la metodología descrita por Shaner \& Finney (1977) y aplicando la siguiente ecuación:

$$
A B C D E=\sum_{i=1}^{N_{i}-1} \frac{\left(y_{i}+y_{i+1}\right)}{2}\left(t_{i+1}-t_{i}\right)
$$

En donde: $Y_{i}=$ severidad inicial; $Y_{i+1}=$ Severidad actual; $\mathrm{t}_{\mathrm{i}+1}=$ tiempo actual; $\mathrm{t}_{\mathrm{i}}=$ tiempo inicial.

Variables climáticas. Se tomaron los datos climáticos diarios de la estación meteorológica, que se encuentra en la granja Montelindo, para correlacionarlos con la severidad del tizón tardío. Se registraron datos de precipitación acumulada cada cuatro días, con ayuda de un pluviógrafo; humedad relativa promedio cada cuatro días (HRP), con un termohigrógrafo y temperatura diaria, con termómetros de máxima, media y mínima.

\section{RESULTADOS Y DISCUSIÓN}

Severidad (\%) del tizón tardío en los dos sistemas de producción. Los sistemas productivos a libre exposición y bajo semitecho a los 25 días después de inoculación, no mostraban diferencias estadísticas $(P=0,68)$, en los valores de severidad con 31 y $30 \%$, respectivamente. Desde esa fecha en 
adelante, la severidad del tizón tardío en el sistema productivo a libre exposición siempre fue mayor que la del sistema productivo bajo semitecho, en todas las demás evaluaciones, terminando a los 49 días después de la inoculación, con una severidad de $47 \%$, en el sistema productivo bajo semitecho y de $77 \%$, en el sistema productivo a libre exposición, con altas diferencias estadísticas, entre los dos sistemas productivos $(P<0,0001)$. Esto explica, la función de la cubierta tipo semitecho, la cual, evitó el contacto directo de la lluvia continua con las plantas, disminuyendo la severidad de la enfermedad, sabiendo que patógenos, como Ph. infestans, liberan zoosporas en el agua, presente sobre las superficies foliares, facilitando así la dispersión del patógeno y el incremento de la enfermedad (Castaño-Zapata, 2002).

Tasa de desarrollo ( $\mathbf{r}$ ) del tizón tardío en los dos sistemas de producción. La tasa de desarrollo del tizón tardío mostró dos tendencias de avance de la enfermedad: La primera, fue la tasa de desarrollo en el sistema de producción a libre exposición, que tuvo un valor de $\mathbf{r}=0,15$ unidades por día; la segunda, corresponde al sistema de producción bajo semitecho, con una $\mathbf{r}=0,09$ unidades por día.

Severidad (\%) del tizón tardío en las introducciones sembradas a libre exposición y factores climáticos. Según Erwin \& Ribeiro (1996), la temperatura mínima para el crecimiento de $P h$. infestans es de $4^{\circ} \mathrm{C}$; la óptima, de $20^{\circ} \mathrm{C}$ y la máxima, de $26^{\circ} \mathrm{C}$. Por otra parte, Jaramillo et al. (2007) indican que la humedad relativa favorable para el desarrollo de la enfermedad debe estar por encima del $80 \%$; estas variables climáticas no mostraron variaciones notables a través del tiempo; se registró una temperatura promedio de 24 a $26^{\circ} \mathrm{C}$, a libre exposición y bajo semitecho, respectivamente y una humedad relativa promedio de $78 \%$, para los dos sistemas productivos. Los valores registrados fueron favorables para el desarrollo del tizón tardío (Figura 1).

La precipitación desempeñó un papel de mayor importancia, ya que en tan solo periodos acumulados de 4 días, pasó de 6 a $114 \mathrm{~mm}$ acumulados, 9 días después de inoculación y $90 \mathrm{~mm}$, a los 13 días, después de inoculación, lo cual, se corrobora siguiendo la teoría del sistema predictivo de BLITECAST, desarrollado en la Universidad Estatal de Pensilvania, a principios de 1970, que interpreta los patrones de precipitación, de temperatura y de alta humedad relativa, sobre el potencial de epidemias del tizón tardío en cultivos de papa y que tiene como base una precipitación de $30 \mathrm{~mm}$ de agua, en 10 días continuos, para que la enfermedad se manifieste (Krause et al. 1975; Mackenzie, 1981).

Es de resaltar que el cultivo se encontraba en la etapa de pleno llenado de fruto, en la cual, simultáneamente, realiza crecimiento vegetativo y floración, factores determinantes para que la enfermedad se manifestara y presentara un rápido crecimiento exponencial, cumpliendo con los componentes del triángulo de la enfermedad, es decir, un ambiente favorable, un patógeno virulento y la diferencia en respuesta, dado por la constitución genética de las introducciones evaluadas. Las plantas del testigo fueron las primeras en manifestar los síntomas de la enfermedad a los 13 días después de la inoculación y, cuatro días más tarde, todas las introducciones tenían valores menores al 15\% de severidad. Después de cada pico de lluvia hubo un incremento de la enfermedad, independientemente de las introducciones (Figura 1).

Todas las introducciones y el testigo comercial, a los 39 días después de la inoculación, superaron la línea roja, que señala el $50 \%$ de severidad de la enfermedad. Es de resaltar que las introducciones LA1480, LA2076 y la introducción IAC445 fueron las que mejor comportamiento mostraron frente al desarrollo de la enfermedad, con valores de severidades finales de 55, 62 y 68\%, respectivamente, pero sin diferencias estadísticas entre las introducciones $(P=0,01)$ (Figura 1$)$.

En general, los resultados de severidad de la enfermedad indicaron una susceptibilidad generalizada de las introducciones evaluadas, lo cual, se puede atribuir, como lo reportan Stintzi et al. (1993), a la evolución que ha tenido el patógeno, a través del tiempo, desarrollando mecanismos para protegerse a sí mismo de enzimas degradantes, como las glucanasas y quitinasas, producidas por las plantas, en respuesta al ataque de patógenos, por medio de proteínas secretoras, que inhiben la actividad enzimática de aquellas, deteniendo la producción de inductores de resistencia (Abramovitch \& Martin, 2004).

Tasa de desarrollo ( $\mathbf{r}$ ) del tizón tardío en las introducciones sembradas a libre exposición. Al igual que la severidad, la tasa de desarrollo de la enfermedad mostró diferencias estadísticas $(P<0,005)$, entre los materiales en ambos sistemas de producción. Al linealizar la severidad de la enfermedad y calculando las tasas de desarrollo, se notó que todas las introducciones y el testigo sobrepasaron la línea, que está demarcando el valor cero (0), la cual, epidemiológicamente, significa el $50 \%$ de severidad. La tasa de desarrollo en este sistema productivo varió entre 0,13 unidades por día, para la introducción LA1480 y el testigo comercial y 0,22 unidades por día, para la introducción IAC412, que tuvo el máximo valor. Introducciones como IAC421 e IAC1688 mostraron mayor susceptibilidad que el testigo, con valores de severidad del 92,94 y $88 \%$, respectivamente y tasas de desarrollo de 0,21, 0,20 y 0,13 unidades por día, respectivamente. Una alta susceptibilidad también se reportó en un estudio realizado por Fry (1978), quien evaluó diferentes variedades de papa, aplicando diferentes dosis de mancozeb contra Ph. infestans, obteniendo en la variedad Hudson, muy susceptible al tizón tardío, una severidad final de $98 \%$ y una $\mathrm{r}=0,28$ unidades por día, sin aplicación del fungicida, en comparación 


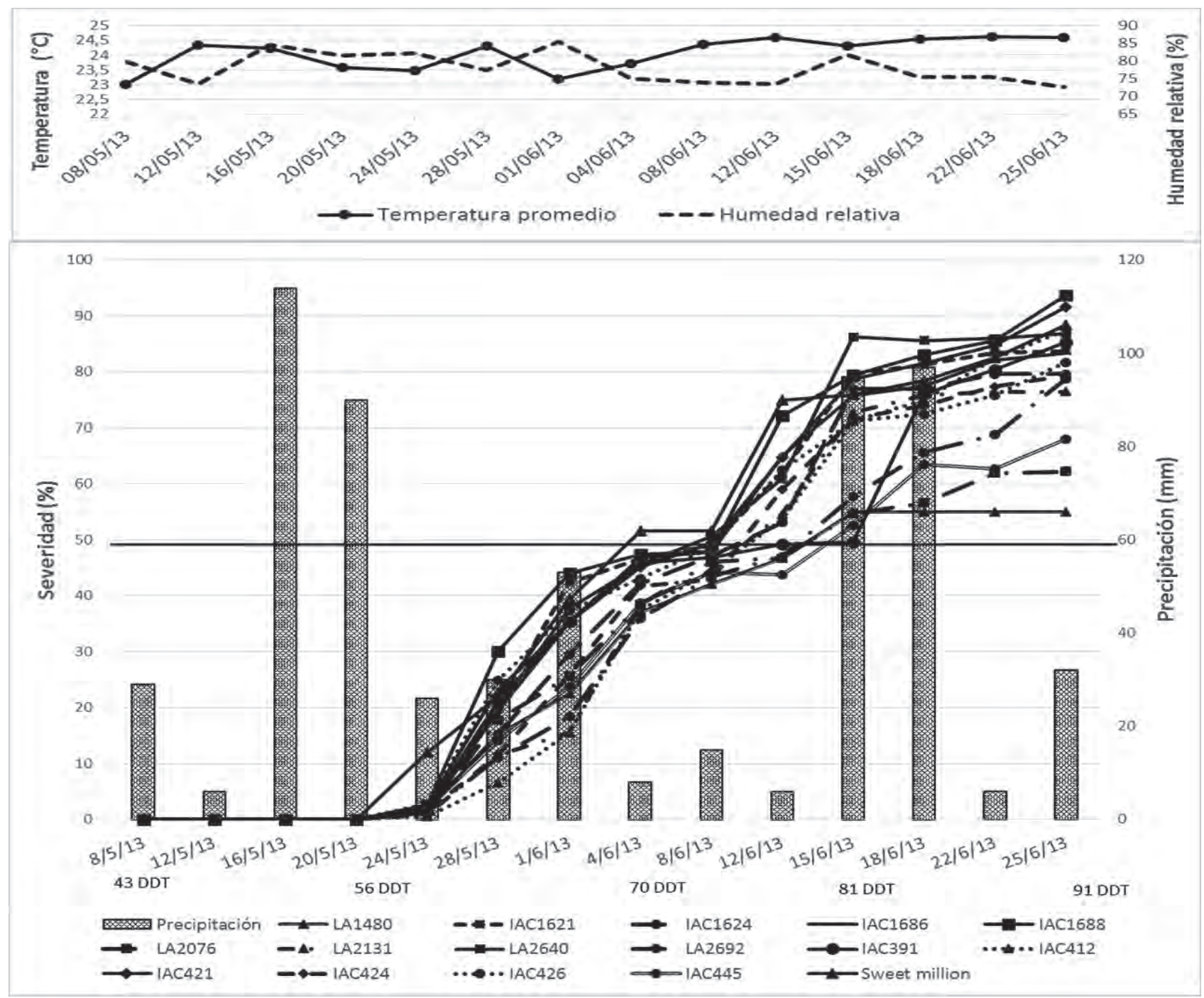

*DDT: Días después de trasplante.

Figura 1. Relaciones entre algunos elementos del clima y la severidad del Tizón tardío, a través del tiempo, en los materiales de tomate tipo cereza, sembradas a libre exposición.

con la aplicación de éste (1,79kg/ha); una severidad final de $35 \%$ y $r=0,18$ unidades por día.

Severidad del tizón tardío en las introducciones sembradas bajo semitecho y factores climáticos. En este caso, 10 de las 15 introducciones evaluadas y el testigo comercial, se mantuvieron por debajo de la línea, que muestra el valor de $50 \%$ de severidad (Figura 2), lo que indica, que el desarrollo lento de la enfermedad, se puede atribuir a la cubierta plástica que se empleó para proteger las plantas de la lluvia directa, la cual, corroborando lo que afirman Tamayo \& Jaramillo (2006), ayuda a que los esporangios de $P$ h. infestans sean fácilmente diseminados. Asimismo, Becktell et al. (2005) comprobaron, en un experimento realizado en tomate bajo invernadero, que el riego dirigido a la superficie del suelo disminuía casi el doble la incidencia y la severidad, $35 \pm$ $8 \%$ y $2 \pm 1 \%$, respectivamente, del tizón tardío, comparado con el riego dirigido sobre el follaje, $68 \pm 12 \%$ y $16 \pm 9 \%$, respectivamente.

Algunas introducciones mostraron tolerancia al avance de la enfermedad, como LA2076, LA2131, IAC1624 y LA1480, con valores de severidades finales de 42, 44, 45 y 47\%, respectivamente. Las severidades de la enfermedad que se observan por debajo de la línea roja, en la figura 2, oscilaron entre 42\%, que corresponde a la introducción LA2076 


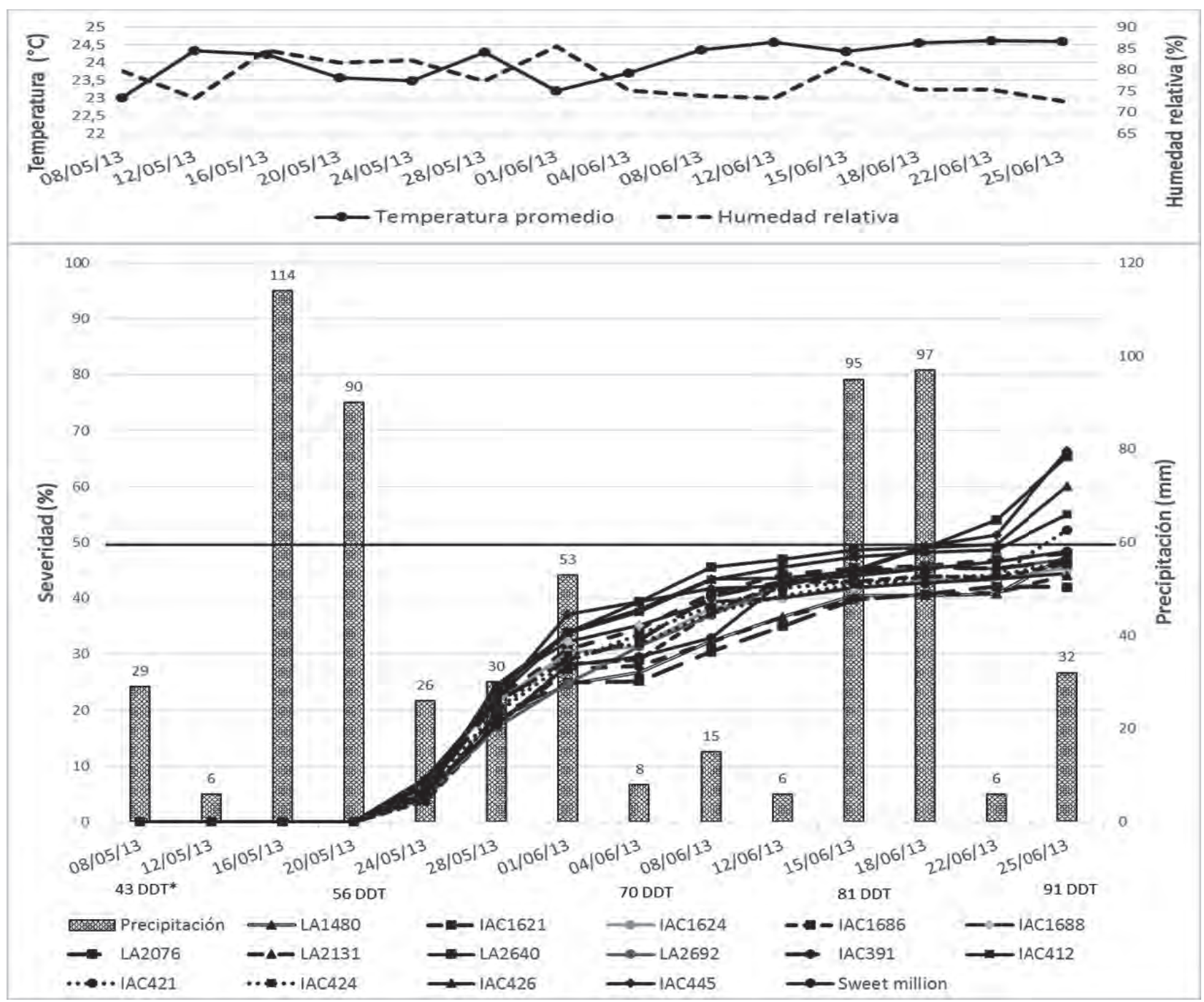

*DDT: Días después de trasplante.

Figura 2. Relaciones entre algunos elementos del clima y la severidad del Tizón tardío, a través del tiempo, en los materiales de tomate tipo cereza, sembradas bajo semitecho.

y $48 \%$, en el testigo comercial; este comportamiento, se observó después de 39 días después de la inoculación (81 DDT), en donde hubo una acumulación de $98 \mathrm{~mm}$ de agua lluvia y mientras que en estos materiales se mantenía estable la severidad de la enfermedad, en las introducciones IAC421, IAC412, IAC426, LA2640 e IAC445 aumentaron los valores, entre 52 y $66 \%$.

Como se observa, la introducción LA2076 mostró un buen comportamiento en los dos sistemas de producción evaluados; la introducción IAC445 tuvo un valor de severidad final de $66 \%$, casi el mismo comportamiento comparado con el sistema de producción a libre exposición, que fue de $68 \%$, en este caso, siendo la introducción más susceptible (Figura 2); no obstante, las introducciones evaluadas mostraron una susceptibilidad generalizada a la enfermedad. De acuerdo con Barbosa et al. (2008), en la búsqueda de resistencia al tizón tardío, se debe evaluar un número considerable de genotipos, debido a que la resistencia es poligénica y de carácter dominante, lo que requiere de una búsqueda intensiva en germoplasma pariente de la especie cultivada, por lo cual, se puede concluir que es necesario continuar con estudios similares a éste, en diferentes áreas geográficas del país, de tal manera que se pueda explorar una mayor cantidad de genotipos y poblaciones del patógeno y así ampliar la posibilidad de encontrar resistencia a la enfermedad. 
Tasa de desarrollo ( $\mathbf{r}$ ) del tizón tardío en las introducciones sembradas bajo semitecho. Los materiales mostraron una disminución numérica considerable en la tasa de desarrollo de la enfermedad; el valor de $r$ más bajo fue 0,07 unidades por día, para la introducción IAC1624 y el valor más alto fue 0,10 unidades por día, para las introducciones IAC1686, LA2640, IAC412, IAC421, IAC426 e IAC445, valores menores a los alcanzados a libre exposición.

Se observaron diferencias numéricas entre los dos sistemas de producción, entre 0,06 unidades por día, para los valores mínimos, hasta 0,12 unidades por día, para los valores máximos de tasas de desarrollo de la enfermedad (r). El caso más notable fue el de la introducción IAC1624, que pasó de ser una de las que tuvieron un valor alto de 0,18 unidades por día, a libre exposición, a tener el valor más bajo de r, en condiciones semi-controladas, de 0,07 unidades por día, lo que indica una alta interacción genotipo-ambiente; en este caso, el mejoramiento del ambiente, le permite al genotipo expresar mejor su potencial y tener un menor desarrollo de la enfermedad.

El sistema de producción bajo semitecho es adecuado para disminuir todos los parámetros epidemiológicos del tizón tardío, debido, principalmente, al hecho que, en este sistema, no hay un efecto directo de la lluvia sobre las plantas.

Área bajo la curva del desarrollo de la enfermedad (ABCDE). La tabla 1 muestra las $\mathrm{ABCDE}$ de cada introducción respecto a los dos sistemas de producción, comparándolas, a la vez, con las tasas de desarrollo (r) y las severidades finales. Analizando el ABCDE en los dos sistemas productivos, se observa que la intensidad de la enfermedad a libre exposición es mayor que bajo condiciones semi-controladas, con valores promedios de 1.573 y 1.133 unidades, respectivamente. Este comportamiento coincide con los resultados del estudio de Foster \& Hausbeck (2010), quienes evaluaron, bajo dos sistemas de producción -campo abierto e invernadero-, el efecto de los fungicidas fluopicolide y mandipropamida sobre Phytophthora capsici, en dos variedades de pimentón 'Paladin'-resistente y 'Red Knight'-susceptible, observando que sin tratamiento químico, en el sistema de producción a campo abierto, la variedad resistente y la susceptible expresaron el ABCDE más alto, con 963 y 5.494 unidades, respectivamente, comparadas con el sistema productivo invernadero, en donde fueron más bajas, con 64 y 141 unidades, respectivamente, en ambas variedades; sin embargo, los valores del $A B C D E$ de todas las introducciones

Tabla 1. Área bajo la curva del desarrollo de la enfermedad (ABCDE), tasas de desarrollo (r) y severidades (\%) finales, en las 15 introducciones y el testigo comercial (Sweet million), en los dos sistemas de producción.

\begin{tabular}{|c|c|c|c|c|c|c|}
\hline \multirow{2}{*}{ Materiales } & \multicolumn{3}{|c|}{ Libre exposición } & \multicolumn{3}{c|}{ Semitecho } \\
\cline { 2 - 7 } & ABCDE & $\mathrm{r}$ & Severidad final (\%) & ABCDE & $\mathrm{r}$ & Severidad final (\%) \\
\hline LA1480 & $\mathbf{1 . 2 3 6}$ & $\mathbf{0 , 1 3}$ & $\mathbf{5 5}$ & $\mathbf{1 . 0 0 2}$ & $\mathbf{0 , 0 9}$ & $\mathbf{4 7}$ \\
\hline IAC1621 & 1.750 & 0,18 & 83 & 1.183 & 0,08 & 48 \\
\hline IAC1624 & 1.337 & 0,18 & 79 & 1.107 & 0,07 & 45 \\
\hline IAC1686 & 1.715 & 0,16 & 83 & $\mathbf{1 . 0 8 2}$ & $\mathbf{0 , 1 0}$ & $\mathbf{4 6}$ \\
\hline IAC1688 & 1.859 & 0,20 & 94 & 1.136 & 0,08 & 44 \\
\hline LA2076 & $\mathbf{1 . 3 1 8}$ & $\mathbf{0 , 1 5}$ & $\mathbf{6 2}$ & $\mathbf{9 7 7}$ & $\mathbf{0 , 0 9}$ & $\mathbf{4 2}$ \\
\hline LA2131 & 1.472 & 0,18 & 76 & 998 & $\mathbf{0 , 0 9}$ & $\mathbf{4 4}$ \\
\hline LA2640 & 1.782 & 0,18 & 87 & 1.311 & 0,10 & 65 \\
\hline LA2692 & 1.590 & 0,17 & 80 & $\mathbf{1 . 0 9 2}$ & $\mathbf{0 , 0 9}$ & $\mathbf{4 7}$ \\
\hline IAC391 & 1.555 & 0,18 & 85 & 1.148 & 0,08 & 47 \\
\hline IAC412 & 1.479 & 0,22 & 88 & 1.252 & 0,10 & 55 \\
\hline IAC421 & 1.741 & 0,21 & 92 & 1.115 & 0,10 & 52 \\
\hline IAC424 & 1.530 & 0,17 & 79 & 1.102 & 0,09 & 46 \\
\hline IAC426 & 1.660 & 0,16 & 82 & 1.255 & 0,10 & 60 \\
\hline IAC445 & 1.313 & 0,15 & 68 & 1.274 & 0,10 & 66 \\
\hline Testigo (Sweet & 1.825 & 0,13 & 88 & 1.087 & 0,09 & 68 \\
\hline million) & $\mathbf{1 . 5 7 3}$ & $\mathbf{0 , 1 7}$ & $\mathbf{8 0}$ & $\mathbf{1 . 1 3 3}$ & $\mathbf{0 , 0 9 1}$ & $\mathbf{5 1}$ \\
\hline Promedio & & & & & \\
\hline
\end{tabular}


son altos, lo que indica una susceptibilidad generalizada de los materiales de tomate evaluados. Byrne et al. (1997), al evaluar las cultivariedades de tomate Ohio 8245 y 7814, en Michigan, Indiana, Estados Unidos, contra Ph. infestans, hallaron valores de $A B C D E$ que oscilaron entre 1.154 y 2.314 unidades, respectivamente, similares y aún superiores a los obtenidos en este estudio; indicando que estas cultivariedades fueron altamente susceptibles a $P$ h. infestans.

Las introducciones LA1480 y LA2076 fueron las que mejor respuesta expusieron respecto al ataque del patógeno, mostrando algún grado de tolerancia y evidenciando menos intensidad de la enfermedad con el tiempo, en los dos sistemas de producción.

La introducción LA1480 en el sistema a libre exposición fue la que menos intensidad de la enfermedad mostró, con un ABCDE de 1.236 unidades; analizada en el sistema bajo semitecho, se observó una reducción de los valores acumulados de $A B C D E$, teniendo como resultado final, una diferencia en el ABDCE de 234 unidades (Tabla 1). En la evaluación realizada el 28 de mayo de 2013 (22 días después de inoculación), a libre exposición, se observaron valores de severidad promedios, cercanos al $20 \%$, pero para las evaluaciones entre el 01 de junio de 2013 al 25 de junio de 2013 ( 25 y 49 días después de inoculación), los valores promedios de severidad aumentaron del 30 al 55\% (Figura 2), mientras que en las mismas fechas, los valores promedios de severidad bajo semitecho, oscilaron entre 26 y $47 \%$ (Figura 2). El ABCDE fue uno de los más bajos en ambos sistemas productivos, ya que los valores acumulados de severidad, a través del tiempo, no superaron el 55\% (Tabla 1).

La introducción LA2076 presentó características similares, observándose su mejor respuesta en el sistema de producción bajo semitecho, con una diferencia entre los dos sistemas de producción en el ABDCE, de 341 (Tabla 1). En los sistemas de producción a libre exposición y bajo semitecho, se observó un comportamiento similar en los valores promedios de severidad, de un poco más del $20 \%$, para el 28 de mayo de 2013 (Figuras 1 y 2). En el sistema a libre exposición, en la evaluación realizada el día 01 de junio de 2013 (25 días después de inoculación), los valores promedios de severidad ya sobrepasaban el $30 \%$ y para el día 25 de junio de 2013, llegaban a más de 60\% (Figura 1); es por esto que la intensidad de la enfermedad llegó a valores de ABCDE de 1.318 unidades, mientras que en el sistema bajo semitecho, para los mismos días evaluados, estos valores no superaron el $30 \%$ y finalizaron con $42 \%$ (Figura 2).

Se observaron introducciones que, al pasarlas de libre exposición a semitecho, cambiaron los resultados respecto al ABCDE; este es el caso de las introducciones IAC1686, LA2131 y LA2692, que mejoraron su respuesta a la enfermedad, con diferencias en el ABCDE, de 633, 474 y 498, respectivamente (Tabla 1).

Existe una relación directa entre los parámetros epidemiológicos evaluados: severidad, tasa de desarrollo y área bajo la curva del desarrollo de la enfermedad; cualquiera de estos parámetros es suficiente para analizar desarrollo del tizón tardío y lleva a la misma conclusión.

Conflictos de intereses: El manuscrito fue preparado y revisado con la participación de los autores, quienes declaramos que no existe conflicto de intereses que ponga en riesgo la validez de los resultados presentados

\section{BIBLIOGRAFÍA}

1. ABRAMOVITCH, R.B.; MARTIN, G.B. 2004. Strategies used by bacterial pathogens to suppress plant defenses. Current Opinion Plant Biology. (USA). 7(4):356364.

2. ARNESON, P.A. 2001. Epidemiología de las Enfermedades de las Plantas: Los Aspectos Temporales. The Plant Health Instructor. DOI: 10.1094/ PHI-A-2001-0524-01. Disponible desde Internet en: http://www.apsnet.org/edcenter/advanced/topics/Epidemiologia/Pages/default.aspx (con acceso 30/03/16.

3. BARBOSA, A.F.; HENRIQUES, D.J.; CRUZ, C.D.; SEITI, G.E. 2008. Inheritance of resistance to Phytophthora infestans (Peronosporales, Pythiaceae) in a new source of resistance in tomato [Solanum sp. (formerly Lycopersicon sp.), Solanales, Solanaceae]. Genetics Molec. Biol. Brazil. 31(2):493-497.

4. BECKTELL, M.C.; DAUGHTREY, M.L.; FRY, W.E. 2005. Epidemiology and management of petunia and tomato late blight in the greenhouse. Plant Disease. (USA). 89(9):1000-1008.

5. BYRNE, J.M.; HAUSBECK, M.K.; LATIN, R.X. 1997. Efficacy and economics of management strategies to control anthracnose fruit rot in processing tomatoes in the Midwest. Plant Disease. 81(10):1167-1172.

6. DANE- ENA. 2014. Encuesta Nacional Agropecuaria Ena 2014. Colombia. Disponible desde Internet en: http://207.239.251.112/www/htm3b/public/ena/ ENA_2014.pdf (con acceso 14/02/2016).

7. CASTAÑO-ZAPATA, J. 2002. Principios básicos de fitoepidemiología. Centro Editorial Universidad de Caldas. Manizales, Colombia. 396p. 
8. CHAVARRO, E.; ÁNGEL, J.E. 2005. Reacción en cadena de la polimerasa $16 S$ ADNr y amplificación aleatoria de polimorfismos de ADN para la detección y diferenciación molecular de Ralstonia solanacearum en plantas de Musa sp. Memorias II Seminario Internacional Sobre Producción, comercialización e industrialización de plátano. Manizales. Colombia. p.14-31.

9. COLON, L.T.; NIELSEN, B.J.; DARSOW, U. 2004. Eucablight protocol-Field test for foliage blight resistance (www.eucablight.org).

10. ERWIN, D.; RIBEIRO, O. 1996. Phytophthora diseases worldwide, Ed. APS Press, St. Paul, Minnesota. USA. p.3-6; 168-169, 347, 592.

11. FEDERACIÓN NACIONAL DE CAFETEROS DE COLOMBIA -FNC- \& CENTRO DE INVESTIGACIONES DE CAFÉ -CENICAFÉ-. 2010. Anuario Meteorológico Cafetero 2008. Chinchiná, Caldas, Colombia.

12. FOSTER, J.M.; HAUSBECK, M.K. 2010. Managing Phytophthora crown and root rot in bell pepper using fungicides and host resistance. Plant Disease. 94:697-702.

13. FRY, W.E. 1978. Quantification of general resistance of potato cultivars and fungicide effects for integrated control of potato late blight. Phytopathology. (USA). 68:1650-1655.

14. KOENIG, D.; JIMÉNEZ-GÓMEZ, J.M.; KIMURA, S.; FULOP, D.; CHITWOOD, D.; HEADLAND, L.; KUMAR, R.; COVINGTON, M.; DEVISETTY, U.K.; TATA A.V.; TOHGE, T.; BOLGER, A.; SCHNEEBERGER, K.; OSSOWSKI, S.; LANZ, C.; XIONG, G.; TAYLORTEEPLES, M.; BRADY, S.M.; PAULY, M.; WEIGEL, D.; USADEL, B.; FERNIE, A.R.; PENG, J.; SINHA, N.R.; MALOOF, J.N. 2013. Comparative transcriptomics reveals patterns of selection in domesticated and wild tomato. Plant Biology. 110(28):2655-2662.

15. KOGAN, M. 1990. La resistencia de la planta en el manejo de plagas. En: Metcalf, R.L.; Luckman, W.H. (Eds.). Introducción al Manejo Integrado de Plagas. Ed. Limusa. México. 710p.

16. KRAUSE, R.A.; MASSIE, L.B.; HYRE, R.A. 1975. Blitecast, a computarized forecast of potato late blight. Plant Disease Rep. 59:95-98.

17. JAMES, W.C.; CALLBECK, L.C.; HODGSON, W.A.; $\mathrm{SHIH}, \mathrm{C.S} .1971$. Evaluation of a method used to es- timate loss in yield of potatoes caused by late blight. Phytopathology. 61:1471-1476.

18. JARAMILLO, J.; RODRÍGUEZ, V.P.; GUZMÁN, M.; ZAPATA, M.; RENGIFO, T. 2007. Manejo fitosanitario. Protección de cultivos. En: Jaramillo, J.; Rodríguez, V.P.; Guzmán, M.; Zapata, M. \& Rengifo, T. 2007. Manual Técnico de Buenas Prácticas Agrícolas - BPA en la producción de tomate bajo condiciones protegidas. CORPOICA - MANA - Gobernación de Antioquia Centro de Investigación La Selva. FAO. Colombia. p.179-180; 199-106; 219-224.

19. LANE, A. 2006. Parientes silvestres de cultivos. Bioversity International. Roma, Italia. 24p. Disponible desde Internet en: http://www.bioversityinternational. org/uploads/tx_news/Parientes_silvestres_de_cultivos_1221.pdf (con acceso 20/06/2015).

20. LEMUS, Y. 2009. Genética de la resistencia a las enfermedades en plantas hortícolas. México. Temas Ciencia y Tecnología. 13(39):41-44.

21. MACKENZIE, D. 1981. Scheduling fungicide applications for potato late blight. USA. Plant Disease. 65(5):394399.

22. NOWICKI, M.; FOOLAD, M.R.; NOWAKOWSKA, M.; KOZIK, E.U. 2012. Potato and tomato late blight caused by Phytophthora infestans: An overview of pathology and resistance breeding. USA. Plant Disease. 96(1):4-17.

23. PIETKIEWICZ, J.B. 1972. Badania odporności ziemniaka na zarazę ziemniaczaną (Phytophthora infestans (Mont.) de Bary) na odciętych liściach. Biuletyn Instytutu Ziemiaka. 9:17-32.

24. SHANER, E.; FINNEY, R.E. 1977. The effect of nitrogen fertilization on the expression of slow mildewing resistance in Knox wheat. USA. Phytopathology. 67:1051-1056.

25. STINTZI, A.; HEITZ, T.; PRASAD, V.; WIEDEMANN-MERDINOGLU, S.; KAUFFMANN, S.; GEOFFROY, P.; LEGRAND, M.; FRITIG, B. 1993. Plant pathogenesis-related proteins and their role in defense against pathogens. Biochimie. (France). 75(8):687-706.

26. TAMAYO M., P.J.; JARAMILLO N., J.E. 2006. Enfermedades del tomate, pimentón, ají y berenjena en Colombia. Guía para su diagnóstico y manejo. Manual Técnico CORPOICA, Centro de Investigación La Selva. $161 p$. 
27. TATAROWSKA, B.; FLIS, B.; ZIMNOCH-GUZOWSKA, E. 2011. Biological Stability of Resistance to Phytophthora infestans (Mont.) de Bary in 22 Polish Potato Cultivars Evaluation in Field Experiments. Potato Association of America. Młochów, Poland. Pp 1 - 9.
28. VAN DER PLANK, J.E. 1963. Plant diseases. Epidemics and control. Academic Press, New York. 349p.

Recibido: Noviembre 24 de 2015

Aceptado: Abril 1 de 2016

\section{Cómo citar:}

Cardona-Piedrahita, L.F.; Castaño-Zapata, J.; Ceballos-Aguirre, N. 2016. Epidemiología del tizón tardío [Phytophthora infestans (Mont.) de Bary] en quince introducciones de tomate silvestre. Rev. U.D.C.A Act. \& Div. Cient. 19(1): 45-54. 